\title{
Epitaxial growth of silicon and germanium on (100)-oriented crystalline substrates by RF PECVD at $175^{\circ} \mathrm{C}$
}

\author{
M. Labrune ${ }^{1,2, \text { a }}$, X. Bril ${ }^{1}$, G. Patriarche ${ }^{3}$, L. Largeau ${ }^{3}$, O. Mauguin ${ }^{3}$, and P. Roca i Cabarrocas ${ }^{1}$ \\ 1 LPICM, CNRS-École Polytechnique, 91128 Palaiseau Cedex, France \\ 2 TOTAL S.A., Gas \& Power, R\&D Division, Courbevoie, France \\ ${ }^{3}$ Laboratoire de Photonique et de Nanostructures, CNRS, Marcoussis, France
}

Received: 25 January 2012 / Accepted: 14 June 2012

Published online: 13 November 2012

\begin{abstract}
We report on the epitaxial growth of crystalline Si and Ge thin films by standard radio frequency plasma enhanced chemical vapor deposition at $175{ }^{\circ} \mathrm{C}$ on (100)-oriented silicon substrates. We also demonstrate the epitaxial growth of silicon films on epitaxially grown germanium layers so that multilayer samples sustaining epitaxy could be produced. We used spectroscopic ellipsometry, Raman spectroscopy, transmission electron microscopy and X-ray diffraction to characterize the structure of the films (amorphous, crystalline). These techniques were found to provide consistent results and provided information on the crystallinity and constraints in such lattice-mismatched structures. These results open the way to multiple quantum-well structures, which have been so far limited to few techniques such as Molecular Beam Epitaxy or MetalOrganic Chemical Vapor Deposition.
\end{abstract}

\section{Introduction}

In the field of solar energy, there is a continuous search for ways to increase the cost-effectiveness of solar cells. This is particularly the case of crystalline silicon solar cells which is the leading technology and covers more than $80 \%$ of the PV market. For this technology to keep its advantage, reducing the thickness of expensive c-Si wafers is mandatory. Various approaches have already been used to produce efficient and thin c-Si solar cells resulting in efficiencies above $22 \%$ for heterojunction c-Si solar cells on a wafer thinned down to $98 \mu \mathrm{m}$ [1]. However this approach still requires to grow ingots and to slice them into wafers. Another way to cut costs is to grow the mono or multi crystalline silicon directly on a foreign substrate, using for instance Chemical Vapor Deposition [2], or on a polycrystalline seed layer obtained by the crystallization of an amorphous silicon layer, using a catalyst in the case of aluminium induced crystallization [3] or in a catalyst-free approach using solid phase crystallization [4]. However, these processes usually lead to relatively low solar cell efficiencies since there is a non-monotonic relationship between grain size and solar cell efficiency that implies that in the case of multicrystalline silicon one should have very large grains, as reported by Bergmann [5].

More recently the epitaxial growth of Si films on c-Si substrates has been achieved by various techniques such as Plasma Enhanced Chemical Vapor Deposition (PECVD) [6], Atmospheric Pressure CVD [7], Inductively

\footnotetext{
${ }^{a}$ e-mail: martin.labrune@mines-paris.org
}

Coupled PECVD [8] as well as by Hot Wire CVD [9]. In most of these cases, except in reference [6], the substrate was kept at a relatively high temperature to favor the epitaxial growth $\left(T \geqslant 700{ }^{\circ} \mathrm{C}\right)$. These high temperature approaches have resulted in solar cell efficiencies of $17 \%$ for a $50 \mu \mathrm{m}$ thick free-standing c-Si base material epitaxially grown on a porous Si substrate before being detached [10], or over $15 \%$ by growing a $20 \mu \mathrm{m}$ thick epitaxial Si base on a seed substrate and also using diffusion processes [11], and about $7 \%$ when growing a $2 \mu \mathrm{m}$ thick epitaxial layer by HWCVD at $700{ }^{\circ} \mathrm{C}[12]$.

Alike, epitaxial growth of Ge films was obtained by PECVD on (100) $\mathrm{NaCl}$ substrates kept at $450{ }^{\circ} \mathrm{C}$ during growth [13] or by Molecular Beam Epitaxy on (100) GaAs [14]. This is of great interest since simulations demonstrated the feasibility of efficient structures combining Si and Ge materials [15].

Previous results obtained in our laboratory have shown that it is possible to obtain epitaxial layers of Ge on (100) gallium arsenide (GaAs) substrates [16] and of Si on (100) silicon substrates [17-19], both by RF PECVD and at substrate temperature as low as $175^{\circ} \mathrm{C}$. By doing so, we have been able to obtain solar cells with an efficiency as high as $7 \%$ for an intrinsic absorber layer of $2.4 \mu \mathrm{m}$ grown at $165{ }^{\circ} \mathrm{C}[19]$.

\section{Experiments}

All the samples of this study were deposited in a multiplasma monochamber reactor PECVD reactor operated at 
EPJ Photovoltaics

Table 1. Process conditions used to grow the epitaxial films of $\mathrm{Si}$ and Ge at $175{ }^{\circ} \mathrm{C}$.

\begin{tabular}{cccccccc}
\hline $\begin{array}{c}\text { Film on } \\
(111) \text { c-Si }\end{array}$ & $\begin{array}{c}\text { Film on } \\
(100) \text { c-Si }\end{array}$ & $\begin{array}{c}\text { Pressure } \\
\text { (Torr) }\end{array}$ & $\begin{array}{c}\mathrm{SiH}_{4} \\
(\mathrm{sccm})\end{array}$ & $\begin{array}{c}\mathrm{GeH}_{4} \text { in } \mathrm{H}_{2} \\
(\mathrm{sccm})\end{array}$ & $\begin{array}{c}\mathrm{H}_{2} \\
(\mathrm{sccm})\end{array}$ & $\begin{array}{c}\mathrm{RF} \text { power } \\
\left(\mathrm{mW} / \mathrm{cm}^{2}\right)\end{array}$ & $\begin{array}{c}\text { Deposition rate } \\
\left(\AA \mathrm{s}^{-1}\right)\end{array}$ \\
\hline$\mu \mathrm{c}-\mathrm{Ge}: \mathrm{H}$ & Epitaxial & 1.4 & 0 & 5 & 200 & 31 & 0.15 \\
pm-Si:H & Epitaxial & 1.2 & 12 & 0 & 200 & 25 & 0.5 \\
\hline
\end{tabular}

a frequency of $13.56 \mathrm{MHz}$ [20]. We used (100) and (111)oriented Si substrates and (100)-oriented GaAs substrates for the TEM experiments. All the crystalline substrates were submitted to a $30 \mathrm{~s}$ dip in hydrofluoric acid, to remove the native oxide, prior to being loaded in the reactor, which was pumped down to a base pressure below $7 \times 10^{-7}$ Torr. We emphasize that this procedure allows us to achieve heterojonction solar cells with efficiencies up to $17 \%$ [18] and even homojonction solar cells when the deposited layer is epitaxial [17]. This is a good indication of the excellent surface passivation and of the high quality of the epitaxial doped layers respectively, when we use this cleaning procedure and this PECVD reactor. The depositions were performed using silane $\left(\mathrm{SiH}_{4}\right)$ and hydrogen $\left(\mathrm{H}_{2}\right)$ gas mixtures for the silicon films and $\mathrm{H}_{2}$ and germane $\left(\mathrm{GeH}_{4}, 2 \%\right.$-diluted in $\left.\mathrm{H}_{2}\right)$ gas mixtures for the germanium films. All the samples were characterized via spectroscopic ellipsometry using a phase modulated ellipsometer (UVISEL from HORIBA Jobin-Yvon). The Raman spectrometer used in this work is a DILOR Jobin Yvon XY with a He-Ne laser excitation at $632.8 \mathrm{~nm}$. The TEM microscope is a JEOL $2200 \mathrm{FS}$, being able to operate in the STEM (scanning TEM) or TEM mode. High angle X-ray diffraction and grazing incidence X-ray diffraction measurements have been performed using a Rigaku Smartlab high resolution diffractometer equipped with a $9 \mathrm{~kW}$ rotating anode and a 7 -axes goniometer.

\section{Results}

Previous studies in our laboratory have shown that plasma conditions leading to hydrogenated microcrystalline germanium films on glass can eventually lead to an epitaxial growth when applied on a (100)-oriented GaAs substrate [16]. We obtained the same results for silicon films, for which conditions known to lead to hydrogenated polymorphous silicon (pm-Si:H) on glass lead to epitaxial growth when applied to (100)-oriented Si substrates $[17,18,21]$. Other research groups also found that a rather broad range of experimental parameters would eventually lead to unwanted epitaxial films on (100) Si substrates $[22,23]$. In the case of heterojunction solar cells, this epitaxy is unwanted since this crystalline layer does not provide any surface passivation [22]. However, one can take benefit of this capability of PECVD to grow thick epitaxial layers and use them as the active material in solar cells [19] or as the emitter of c-Si solar cells [17].

Table 1 summarizes the plasma conditions used to achieve epitaxy on (100)-oriented substrates for the Si and Ge films respectively. Figure 1 shows the SE spectra of a multilayer stack co-deposited on (100) GaAs, (100) Si and

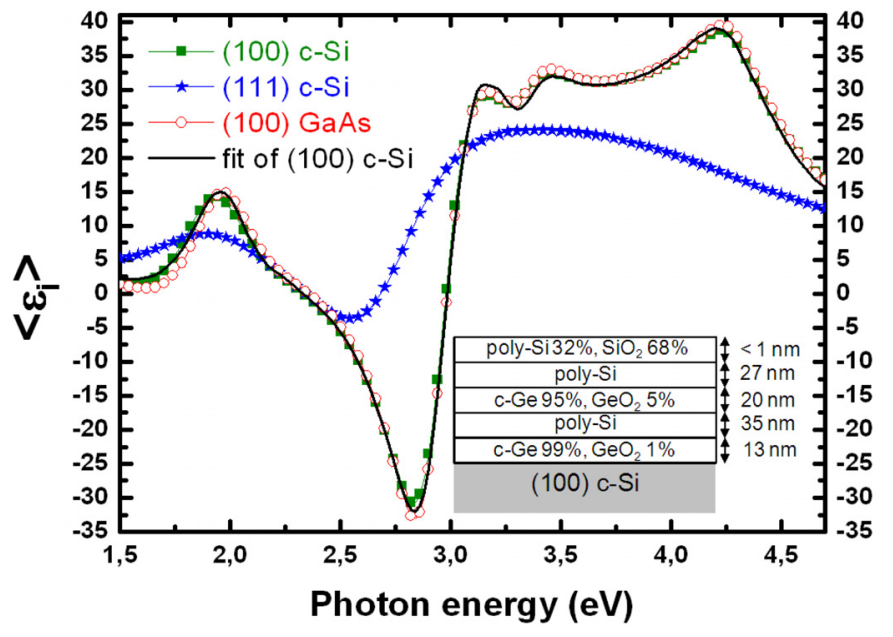

Fig. 1. Imaginary part of the pseudo-dielectric function of the multi layer stack (905071) co-deposited onto various substrates as deduced from SE measurements, the black line corresponds to the fit obtained by modeling the stack deposited on the (100) Si substrate using the optical model described in the inset.

(111) Si. The high photon energy part $(3-5 \mathrm{eV})$ of the SE spectrum is more sensitive to the top and also bulk part of the films. The last deposited layer being silicon, in Figure 1, we can see the characteristic spectrum of c$\mathrm{Si}$, which has two peaks around 3.4 and $4.2 \mathrm{eV}$ on both GaAs and Si (100)-oriented substrates, whereas on (111) c-Si substrate, the silicon films are amorphous (a similar spectrum was measured for the film co-deposited on glass, not shown for clarity). The lower photon energy part of the spectrum is sensitive to the bulk and thickness of the film, the interference fringes providing information on the thickness of the whole stack. The Bruggeman Effective Medium Approximation (BEMA) model [24] used for the film grown on (100) $\mathrm{Si}$ is shown in the inset of Figure 1 and the spectrum obtained from the model is also plotted with a dark line. Interestingly, the measurement performed on the film grown on the (100) GaAs substrate, can be fitted using the same model as the one used to fit the measurement performed on the (100) Si. This means that we obtained the same stack on both (100) substrates. Even though a fit based on crystalline silicon and germanium materials provided a reasonable match with the experimental data (with a figure of merit $\chi^{2}$ of 2.3 ), we could improve the fit $\left(\chi^{2}=1.5\right)$ by using the dielectric function of large grains polysilicon material reference file obtained by Jellison et al. [25] for the silicon layers and a mixture of crystalline germanium and a small fraction of germanium oxide (1-5\%), as obtained by Aspnes and Studna [26] for the germanium layers. There are at least 


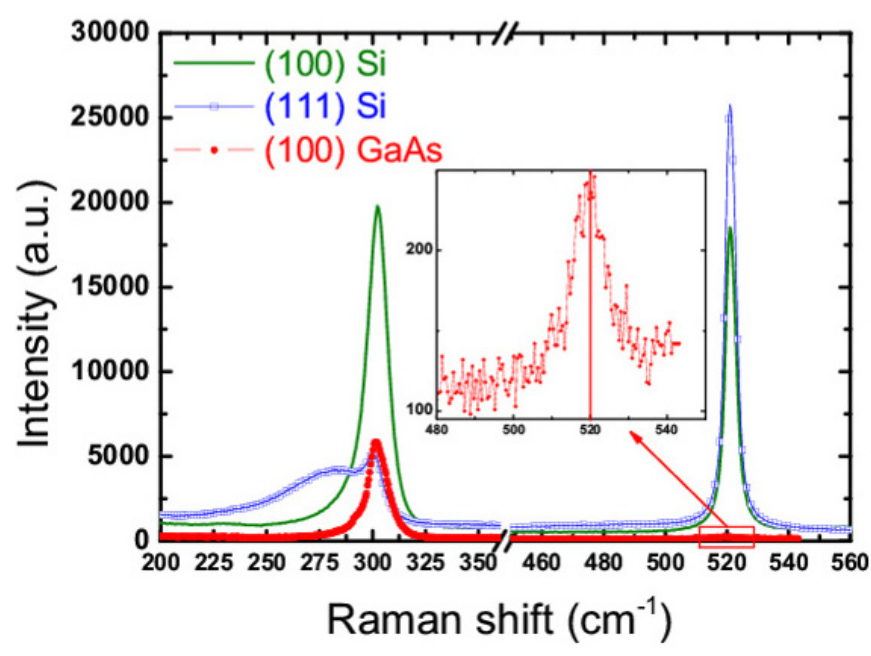

Fig. 2. Raman scattering intensity as a function of the Raman shift for the multi layer samples (905071) co-deposited on various substrates ((100) GaAs, (100) Si and (111) Si).

two reasons that may explain the better fit when considering polycrystalline dielectric function and introducing $\mathrm{GeO}_{2}$ in the Ge layers. The first one is that we cannot expect to have films with no roughness so that among our four interfaces, none is perfectly flat (as shown by the presence of a surface roughness of about $1 \mathrm{~nm}$ in the model of Fig. 1). Introducing a rough interface between each layer could even further reduce $\chi^{2}$ but this is at the cost of drastically increasing the number of parameters of the model. The second one is that these films are produced at $175^{\circ} \mathrm{C}$ in a standard RF PECVD reactor without load-lock nor special precaution concerning gas purity (no gas purifiers) so that we can expect our films to contain carbon and oxygen impurities as well as a significant amount of hydrogen. Those may slightly alter the dielectric functions of the materials as compared to their calibrated crystalline counterparts.

We also investigated the structure of the films by Raman spectroscopy. The Raman spectra of the stack deposited on the three substrates are shown in Figure 2, the substrates being (100) GaAs, (100) Si and (111) Si. The Raman spectra of the multilayer films deposited on GaAs and (100) Si show a sharp peak around $300 \mathrm{~cm}^{-1}$, which is consistent with fully crystallized Ge layers [27, 28]. It has been shown that a peak at $300 \mathrm{~cm}^{-1}$ could also originate from Si substrates [29], but in our case, a comparison between a $\mathrm{Si}$ substrate and a $\mathrm{Si}$ substrate capped with a thin epitaxial Ge layer showed that no signal from the $\mathrm{Si}$ substrate alone at $300 \mathrm{~cm}^{-1}$ was distinguishable whereas a very sharp peak would appear in the presence of this thin Ge layer. On the other hand, the film deposited on (111) c-Si substrate displays a shoulder towards lower wavenumbers, indicating that the film is partially crystallized and contains an amorphous phase, since the hydrogenated amorphous Ge has a TO mode at $278 \mathrm{~cm}^{-1}$ [28]. The peak around $520 \mathrm{~cm}^{-1}$ can be assigned to crystalline silicon [27], but does not give much information on $\mathrm{Si}$ substrates where this peak is due to the substrate and

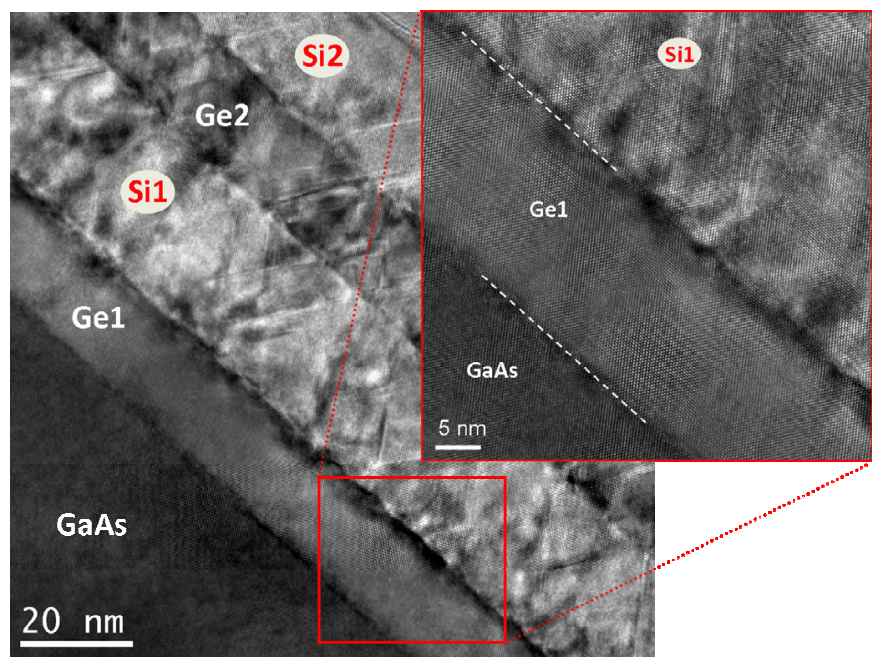

Fig. 3. HRTEM image of the multilayer stack on GaAs. The inset zooms on the two first Ge and Si layers.

masks the small contribution of the amorphous film (at $480 \mathrm{~cm}^{-1}$ ) to the Raman spectrum. However, on the GaAs substrate, we can detect the signal from the c-Si film despite of its small thickness (see inset in Fig. 2). Moreover, one can see that the film is fully crystallized as there is no shoulder at $480 \mathrm{~cm}^{-1}$, as it would be the case if there were an amorphous silicon phase.

We used the sample deposited on (100) GaAs for TEM measurements. In Figure 3, we show an example of the High Resolution TEM (HRTEM) images of the stack we obtained. The red square in Figure 3 indicates the area on which we zoomed in the inset to focus on the two first layers and the two first interfaces. Based on such images, we can get a reasonable estimate of the thickness of each layer. The thicknesses are approximately $20 \mathrm{~nm}$ for the Ge layers and $30 \mathrm{~nm}$ for the Si layers. These results are in good agreement with the ones obtained by SE even though some discrepancy exists for the first Ge layer, the closest layer to the substrate. The fact is that the accuracy of the SE fit is not very sensitive to the value of the thickness of the first Ge layer so that the value we obtained had a significant error bar $(\approx 1.5 \mathrm{~nm})$. On the inset of Figure 3, it appears that they are very few defects in the first Ge layer and that some start to appear at the interface between the first silicon layer and the first germanium layer. In all these layers we could observe some dislocations but it must be noted that that they do not propagate systematically from one layer to the other and that these dislocations do not prevent our layers to keep a monocrystalline structure, which supports the data obtained from spectroscopic ellipsometry and Raman spectroscopy.

Moreover, the microscope could also be operated in the Scanning TEM mode and by doing so we could obtain High Angle Annular Dark Field (HAADF) images of our sample as the one we show in Figure 4. For such images, the contrast in the gray scale comes from a difference in the $Z$ value of the elements since we only collect the electrons that have scattered to high deviation angles and therefore the ones that are the most sensitive to the 
EPJ Photovoltaics

Table 2. Spacings between adjacent lattices and calculated lattice parameters obtained by XRD for Si and Ge.

\begin{tabular}{ccccc}
\hline $\begin{array}{c}\text { Diffraction } \\
\text { planes }\end{array}$ & $\begin{array}{c}\text { Distance between } \\
\text { Si planes }(\AA)\end{array}$ & $\begin{array}{c}\text { Si lattice parameter } \\
(\AA)\end{array}$ & $\begin{array}{c}\text { Distance between } \\
\text { Ge planes }(\AA)\end{array}$ & $\begin{array}{c}\text { Ge lattice parameter } \\
(\AA)\end{array}$ \\
\hline 220 & $1.9183 \pm 0.001$ & $a_{\|}=5.4257 \pm 0.003$ & $1.97974 \pm 0.001$ & $a_{\|}=5.5996 \pm 0.003$ \\
400 & $1.358 \pm 0.001$ & $a_{\|}=5.432 \pm 0.004$ & - & $a_{\perp}<5.65325$ \\
004 & $1.3609 \pm 0.001$ & $a_{\perp}=5.444 \pm 0.004$ & - & $a_{\|}>5.65325$ \\
\hline
\end{tabular}

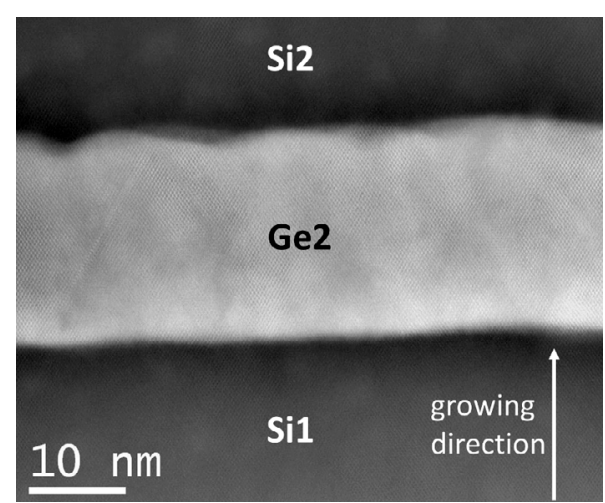

Fig. 4. HAADF image obtained by STEM of the multilayer sample on GaAs.

$Z$ value. This means that we somehow get a "chemical picture" of the stack. Even if the atoms remain in a very crystallized arrangement the elements are not always the same. At the interface between $\mathrm{Si}$ and Ge in Figure 4 we can observe that there is a pronounced "chemical roughness". This means that we do not have perfectly flat interfaces and that we can still have Ge elements in the very first nanometers of the following Si layer. This complements the observation of Figure 3 and can be explained by the deposition technique itself rather than any diffusion process since we used low temperature deposition but we used the same chamber for Ge and Si films growth.

We coupled high angle $2 \theta / \omega$ X-ray diffraction and grazing incidence X-ray diffraction (GIXRD) to study our sample in order to get information about the crystallinity of the films and also to get more quantitative information regarding the lattice parameters. The Rigaku Smartlab diffractometer, based in the LPN laboratory, allowed us to study the diffraction from the crystallographic planes parallel to the surface (lattice parameter: $a_{\perp}$ ) by scanning the $2 \theta$ and $\omega$ angles in the direction normal to the surface. $2 \theta$ is the angle between the diffracted beam and the surface and $\omega$ is the angle between the incident beam and the surface of the sample. We also studied the diffraction of the crystallographic planes perpendicular to the surface (lattice parameter: $a_{\|}$) by scanning $2 \theta \chi$ and $\varphi$ angles in grazing incidence $(\varphi$ corresponds to the rotation of the sample on itself while $2 \theta \chi$ is the sample in-plane angle between the crystallographic planes and the detection). This $2 \theta \chi / \varphi$ in-plane configuration corresponds to a $2 \theta / \omega$ configuration for crystallographic planes normal to the surface. Figure 5a shows the GIXRD of the $\{220\}$ planes of the GaAs substrate and the Ge and Si layers. Knowing the interplanar distance of the 220 GaAs substrate planes, we can deduce the 220 interplanar distance
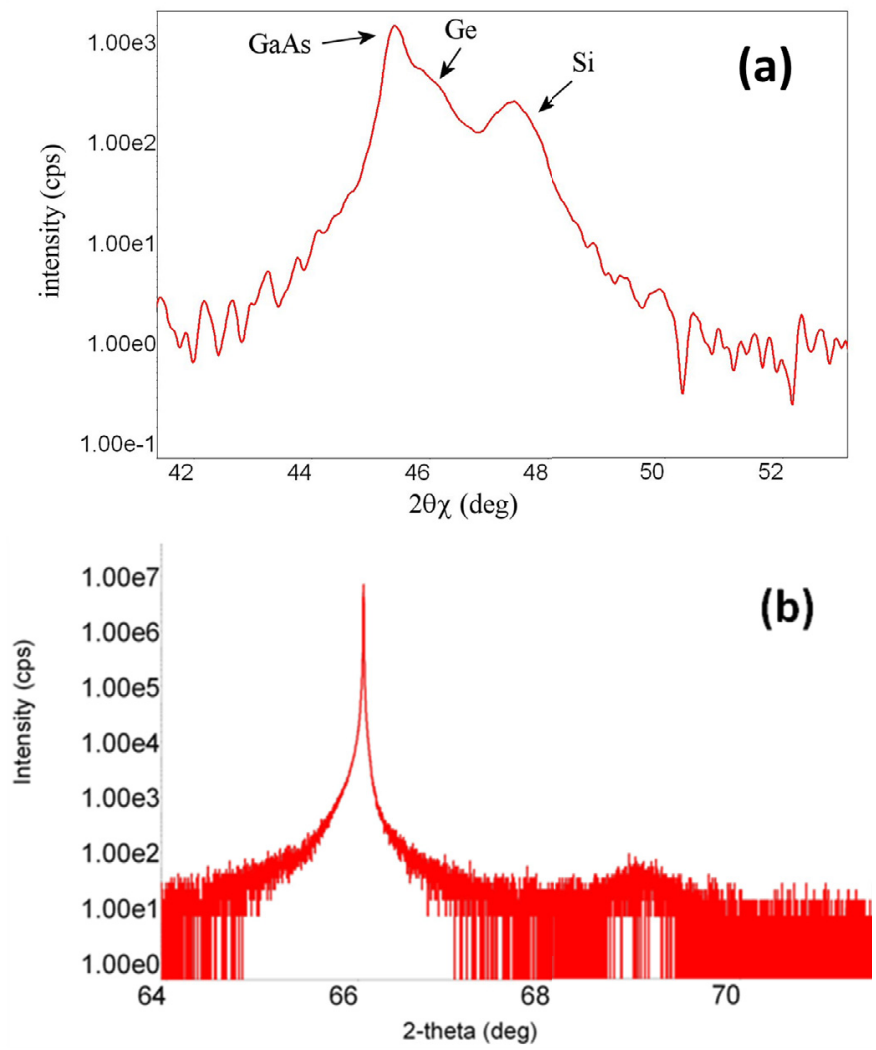

Fig. 5. (a) XRD measurement of the 220 planes perpendicular to the surface of the film. (b) High resolution XRD measurement of the $\{004\}$ planes parallel to the surface of the film.

for Ge and Si by measuring the mismatch between the Ge, $\mathrm{Si}$ and GaAs peaks. These values are summarized in the first row of Table 2 .

We also performed GIXRD of the $\{400\}$ planes normal to the surface of GaAs, Ge and Si (not shown here). This allows us to calculate the distance between planes in the 400 direction for Si (second row of Tab. 2). Unfortunately we were not able to deconvolve the peaks due to GaAs and Ge but based on the fact the graph showed a shoulder towards higher angles around the 400 peak of GaAs, we can assume that we have a lattice parameter lower than the one of GaAs.

Finally we operated the XRD set-up in the high angle $2 \theta / \omega$ configuration in order to obtain the high resolution diffraction from the planes parallel to the surface. We obtained diffraction from the $\{004\}$ planes that we show in Figure 5b. Even though we could not observe a distinct peak for Ge we could still notice the dissymmetry of the GaAs peak towards smaller angles, indicating a Ge $a_{\perp}$ lattice parameter higher than the GaAs $a_{\perp}$ lattice parameter. 
Nevertheless, we could again calculate the parameters for Si and we summarized them in the third row of Table 2.

Based on these results, averaging the value of $a_{\|}$for $\mathrm{Si}$, and using the formula in equation (1) we could calculate the bulk lattice parameter of Si. We used for Si the

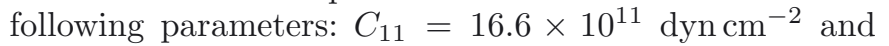
$C_{22}=6.4 \times 10^{11}{\mathrm{dyn} \mathrm{cm}^{-2}}$.

$$
\frac{a_{\perp}-a_{b u l k}}{a_{\text {bulk }}}=-2 \frac{C_{12}}{C_{11}} \frac{a_{\|}-a_{b u l k}}{a_{b u l k}} .
$$

We obtained $a_{\text {bulk }}=5.4374 \pm 0.004 \AA$. This parameter is slightly higher that the theoretical value of $5.431 \AA$. Furthermore, we measure $a_{\|}<a_{\perp}$ indicating a slight compressive constraint for $\mathrm{Si}$.

For Ge, $a_{\perp}>5.65325 \AA$ (GaAs lattice parameter) and $a_{\|}=5.5996 \pm 0.003 \AA$, that corresponds to an important compressive strain. It is so far rather speculative to explain these results but we expect the process conditions to play a major role on the constraints of the material. Indeed, in the case of Ge, the layers were deposited from $\mathrm{GeH}_{4}$ which was $2 \%$-diluted in $\mathrm{H}_{2}$ so that the plasma was mostly a hydrogen plasma resulting in much more aggressive deposition conditions compared to a-Si:H deposition case.

\section{Conclusions}

In this paper we have demonstrated the epitaxial nature of Si and Ge films grown on (100) crystalline Si as well as the growth of $\mathrm{Si}$ on epitaxially grown Ge layers in a PECVD reactor at $175^{\circ} \mathrm{C}$. Raman spectroscopy, Transmission Electron Microscopy, X-ray diffraction and Spectroscopic Ellipsometry consistently showed that the films were made up of monocrystalline layers. Further studies are needed to get a better understanding of the growth mechanisms and also to study the influence of the lattice parameter of the substrate on the constraints in the resulting films.

\section{References}

1. T. Mishima, M. Taguchi, H. Sakata, E. Maruyama, Sol. Energy Mater. Sol. Cells 95, 18 (2011)

2. T. Yamazaki, Y. Uraoka, Takashi Fuyuki, Thin Solid Films 487, 26 (2005)

3. W. Fuhs, S. Gall, B. Rau, M. Schmidt, J. Schneider, Sol. Energy 77, 961 (2004)

4. P.A. Basore, Proceedings of the 4 th World Conference on Photovoltaic Energy Conversion, Waikoloa, Hawaii, 2006
5. R.B. Bergmann, Appl. Phys. A 69, 187 (1999)

6. R. Shimokawa, M. Yamanaka, I. Sakata, Jpn J. Appl. Phys. 46, 7612 (2007)

7. E. Schmich, N. Schillinger, S. Reber, Surf. Coat. Technol. 201, 9325 (2007)

8. M. Kambara, H. Yagi, M. Sawayanagi, T. Yoshida, J. Appl. Phys. 99, 074901 (2006)

9. H.M. Branz, C.W. Teplin, D.L. Young, M.R. Page, E. Iwaniczko, L. Roybal, R. Bauer, A.H. Mahan, Y. Xu, P. Stradins, T. Wang, Q. Wang, Thin Solid Films 516, 743 (2008)

10. J.H. Petermann, D. Zielke, J. Schmidt, F. Haase, E.G. Rojas, R. Brendel, Prog. Photovolt.: Res. Appl. 20, 1 (2012)

11. I. Kuzma-Filipek, K.V. Nieuwenhuysen, J.V. Hoeymissen, M.R. Payo, E.V. Kerschaver, J. Poortmans, R. Mertens, G. Beaucarne, E. Schmich, S. Lindekugel, S. Reber, Prog. Photovolt.: Res. Appl. 18, 137 (2010)

12. K. Alberi, I.T. Martin, M. Shub, C.W. Teplin, M.J. Romero, R.C. Reedy, E. Iwaniczko, A. Duda, P. Stradins, H.M. Branz, D.L. Young, Appl. Phys. Lett. 96, 073502 (2010)

13. R.A. Outlaw, P. Hopson Jr., J. Appl. Phys. 55, 6 (1984)

14. B. Jenichen, V.M. Kaganer, R. Shayduk, W. Braun, A. Trampert, Phys. Stat. Sol. A 206, 1740 (2009)

15. C.-H. Lin, Thin Solid Films 518, S255 (2010)

16. E.V. Johnson, G. Patriarche, P. Roca i Cabarrocas, Appl. Phys. Lett. 92, 103108 (2006)

17. M. Labrune, M. Moreno, P. Roca i Cabarrocas, Thin Solid Films 518, 2528 (2010)

18. J. Damon-Lacoste, P. Roca i Cabarrocas, J. Appl. Phys. 105, 063712 (2009)

19. R. Cariou, M. Labrune, P. Roca i Cabarrocas, Sol. Energy Mater. Sol. Cells 95, 2260 (2011)

20. P. Roca i Cabarrocas, J.B. Chévrier, J. Huc, A. Lloret, J.Y. Parey, J.P.M. Schmitt, J. Vac. Sci. Technol. A 9, 2331 (1991)

21. P. Roca i Cabarrocas, R. Cariou, M. Labrune, J. NonCryst. Solids 358, 2000 (2012)

22. J.J.H. Gielis, P.J. van den Oever, B. Hoex, M.C.M. van de Sanden, W.M.M. Kessels, Phys. Rev. B 77, 205329 (2008)

23. C.W. Teplin, D.H. Levi, E. Iwaniczko, K.M. Jones, J.D. Perkins, H.M. Branz, J. Appl. Phys. Lett. 97, 103536 (2005)

24. D.A.G. Bruggeman, Ann. Phys. 416, 636 (1935)

25. G.E. Jellison Jr., F. Chisholm, S.M. Gorbatkin, Appl. Phys. Lett. 62, 25 (1993)

26. D.E. Aspnes, A.A. Studna, Phys. Rev. B 27, 985 (1983)

27. J.H. Parker Jr., D.W. Feldman, M. Ashkin, Phys. Rev. B 155, 3 (1967)

28. D. Bermejo, M. Cardona, J. Non-Cryst. Solids 32, 405 (1979)

29. A.V. Kolobov, J. Appl. Phys. 87, 2926 (1999) 